\title{
Multi-frequency observations of SNR J0453-6829 in the LMC
}

\section{A composite supernova remnant with a pulsar wind nebula}

\author{
F. Haberl ${ }^{1}$, M. D. Filipović ${ }^{\text {, L. M. Bozzetto }}$, E. J. Crawford ${ }^{2}$, S. D. Points ${ }^{3}$, W. Pietsch ${ }^{1}$, A. Y. De Horta ${ }^{2}$, N. Tothill ${ }^{2}$, \\ J. L. Payne ${ }^{2}$, and M. Sasaki ${ }^{4}$
}

\author{
1 Max-Planck-Institut für extraterrestrische Physik, Giessenbachstraße, 85748 Garching, Germany \\ e-mail: fwh@mpe.mpg.de \\ 2 University of Western Sydney, Locked Bag 1797, Penrith South DC, NSW1797, Australia \\ 3 Cerro Tololo Inter-American Observatory, National Optical Astronomy Observatory, Cassilla 603, La Serena, Chile \\ ${ }^{4}$ Institut für Astronomie und Astrophysik Tübingen, Sand 1, 72076 Tübingen, Germany
}

Received 6 February 2012 / Accepted 21 June 2012

\section{ABSTRACT}

\begin{abstract}
Context. The Large Magellanic Cloud (LMC) is rich in supernova remnants (SNRs), which can be investigated in detail with radio, optical, and X-ray observations. SNR J0453-6829 is an X-ray and radio-bright remnant in the LMC, within which previous studies revealed the presence of a pulsar wind nebula (PWN), making it one of the most interesting SNRs in the Local Group of galaxies. Aims. We study the emission of SNR J0453-6829 to improve our understanding of its morphology, spectrum, and thus the emission mechanisms in the shell and the PWN of the remnant.

Methods. We obtained new radio data with the Australia Telescope Compact Array and analysed archival XMM-Newton observations of SNR J0453-6829. We studied the morphology of SNR J0453-6829 from radio, optical, and X-ray images and investigated the energy spectra in the different parts of the remnant.

Results. Our radio results confirm that this LMC SNR hosts a typical PWN. The prominent central core of the PWN exhibits a radio spectral index $\alpha_{\text {Core }}$ of $-0.04 \pm 0.04$, while in the rest of the SNR shell the spectral slope is somewhat steeper with $\alpha_{\text {Shell }}=$ $-0.43 \pm 0.01$. We detect regions with a mean polarisation of $P \cong(12 \pm 4) \%$ at $6 \mathrm{~cm}$ and $(9 \pm 2) \%$ at $3 \mathrm{~cm}$. The full remnant is of roughly circular shape with dimensions of $(31 \pm 1) \mathrm{pc} \times(29 \pm 1) \mathrm{pc}$. The spectral analysis of the XMM-Newton EPIC and RGS spectra allowed us to derive physical parameters for the SNR. Somewhat depending on the spectral model, we obtain for the remnant a shock temperature of around $0.2 \mathrm{keV}$ and estimate the dynamical age to 12 000-15000 years. Using a Sedov model we further derive an electron density in the X-ray emitting material of $1.56 \mathrm{~cm}^{-3}$, typical for LMC remnants, a large swept-up mass of $830 M_{\odot}$, and an explosion energy of $7.6 \times 10^{50} \mathrm{erg}$. These parameters indicate a well evolved SNR with an X-ray spectrum dominated by emission from the swept-up material.
\end{abstract}

Key words. ISM: supernova remnants - ISM: individual objects: SNR J0453-6829 - Magellanic Clouds

\section{Introduction}

The Magellanic Clouds (MCs) are considered to be one of the most ideal environments when it comes to the investigation of various supernova remnant (SNR) types and their different evolutionary stages. While their relatively small distance is very favourable for detailed studies, the MCs are also located in one of the coldest parts of the radio sky, allowing us to detect and investigate radio emission with little disturbing Galactic foreground radiation (Haynes et al. 1991). As they are located outside of the Galactic plane, the influence of dust, gas and stars is small, reducing the absorption of soft X-rays. Particularly, the Large Magellanic Cloud (LMC) at a distance of $50 \mathrm{kpc}$ (di Benedetto 2008), allows for detailed analysis of the energetics of various types of remnants. In the radio-continuum regime SNRs are well-known for their strong and predominantly nonthermal radio emission, which is characterised by a typical spectral index of $\alpha \sim-0.5$ (as defined by $S \propto v^{\alpha}$ ). However, this value shows a significant scatter due to the wide variety of SNR types and this variance can be used as an age indicator for the SNR (Filipović et al. 1998a).

SNRs have a significant influence on the structure of the interstellar medium (ISM). The appearance of spherically symmetric shell-like structures is very often perturbed by interaction with a non-homogeneous structure of the ISM. SNRs influence the behaviour, structure and evolution of the ISM. In turn, the evolution of SNRs is dependent on the environment in which they reside.

Here, we report on new radio-continuum and archival X-ray observations of SNR J0453-6829, one of five SNRs in the LMC (B0540-693, N157B, B0532-710, DEML241 and SNR J0453-6829) with a known or candidate pulsar wind nebula (PWN) inside. SNR J0453-6829 was initially classified as an SNR based on the Einstein X-Ray survey by Long et al. (1981, source 1 in their catalogue) and Wang et al. (1991, their source 2). Mathewson et al. (1983) catalogued this object based on studies of optical and MOlonglo Synthesis Telescope (MOST) survey data, and reported an optical size of $140^{\prime \prime} \times 131^{\prime \prime}$. SNR J0453-6829 is also listed in the $408 \mathrm{MHz}$ MC4 catalogue of Clarke et al. (1976) as a distinctive pointlike radio source, for which Mathewson et al. (1983) later remeasured a flux density of $350 \mathrm{mJy}$. Mills et al. (1984) detected this source with specific MOST pointings and reported a rather flat spectral index of $\alpha=-0.38$. ROSAT detected X-ray emission from SNR J0453-6829 during the all-sky survey (Filipović et al. 1998b) and in deeper pointed observations using the PSPC (source 670 in the catalogue of Haberl \& Pietsch 1999) and HRI detector (source 8 in Sasaki et al. 2000). 
Filipović et al. (1998a) added further confirmation from radio data over a wide frequency range. Williams et al. (1999) classified it as an SNR with a "diffuse face". Hughes et al. (1998) presented X-ray spectra based on ASCA observations. Their spectral modelling did not take into account the hard X-ray emission of the PWN, which was discovered later, and therefore overestimated its temperature. The most detailed study of SNR J0453-6829 was performed by Gaensler et al. (2003) based on high resolution radio data obtained in 2002 with the Australia Telescope Compact Array (ATCA) and Chandra X-ray data from 2001. This lead to the discovery of the PWN inside the SNR and an age estimate of around 13000 years. Blair et al. (2006) reported no detection at far ultraviolet wavelengths based on data from the FUSE (Far Ultraviolet Spectroscopic Explorer) satellite. Williams et al. (2006) detected this SNR in their Spitzer IR surveys as the object with the highest $70 \mu \mathrm{m}$ to $24 \mu \mathrm{m}$ flux ratio of any SNR. However, due to high background emission at $70 \mu \mathrm{m}$, in particular in the south western region of the remnant, they could only investigate the northern rim of the SNR shell. No radio pulsar within the area of SNR J0453-6829 was found in the systematic search within the LMC performed by Manchester et al. (2006). Also Payne et al. (2008) did not detect SNR J0453-6829 in their optical spectroscopic survey of LMC SNRs. Finally, Lopez et al. (2009) classified this LMC object based on its circular morphology as a core-collapse SNR.

\section{Observations and data reduction}

\subsection{Radio-continuum}

We observed SNR J0453-6829 with the ATCA on October 5, 1997, with the array configuration EW375 (ATCA Project C634), at wavelengths of $3 \mathrm{~cm}$ and $6 \mathrm{~cm}(8640 \mathrm{MHz}$ and $4800 \mathrm{MHz}$ ). The observations were carried out in the "snapshot" mode, totalling $\sim 1 \mathrm{~h}$ of integration time over a $12 \mathrm{~h}$ period. Source PKS B1934-638 was used for primary calibration and source PKS B0530-727 for secondary calibration. In addition to our own observations, we included unpublished $3 \mathrm{~cm}$ and $6 \mathrm{~cm}$ observations from project C1074 (Gaensler et al. 2003) and mosaic data taken from project C918 (Dickel et al. 2010). When combined together, all $3 \mathrm{~cm}$ and $6 \mathrm{~cm}$ observations of SNR J0453-6829 total $\sim 11 \mathrm{~h}$ of integration time. Baselines formed with the 6th ATCA antenna were excluded, as the other five antennas were arranged in a compact configuration.

The MIRIAD (Sault et al. 1995) and KARMA (Gooch 1996) software packages were used for reduction and analysis. More information on the observing procedure and other sources observed in this session can be found in Bojičić et al. (2007), Crawford et al. (2008b,a, 2010), Čajko et al. (2009), and Bozzetto et al. (2010, 2012a,b). Images were constructed using the MIRIAD multi-frequency synthesis package (Sault \& Wieringa 1994). Deconvolution was achieved with the CLEAN and RESTOR tasks with primary beam correction applied using the LINMOs task. Similar procedures were used for the $U$ and $Q$ stokes parameters.

The ATCA $3 \mathrm{~cm}$ image is shown in Fig. 1 together with $6 \mathrm{~cm}$ contours. We also reanalysed the $20 \mathrm{~cm}$ and $13 \mathrm{~cm}$ observations from Gaensler et al. (2003, ATCA Project C1074) and, while we achieved slightly better resolution and rms noise, the estimated flux densities stay essentially the same (Fig. 1). We measured the flux density of SNR J0453-6829 at six frequencies, which are summarised in Table 1 . For the $36 \mathrm{~cm}(0.843 \mathrm{MHz}$, MOST) measurement we used the unpublished image described by Mills et al. (1984) and for $20 \mathrm{~cm}(1.388 \mathrm{GHz}$, ATCA project C373) the
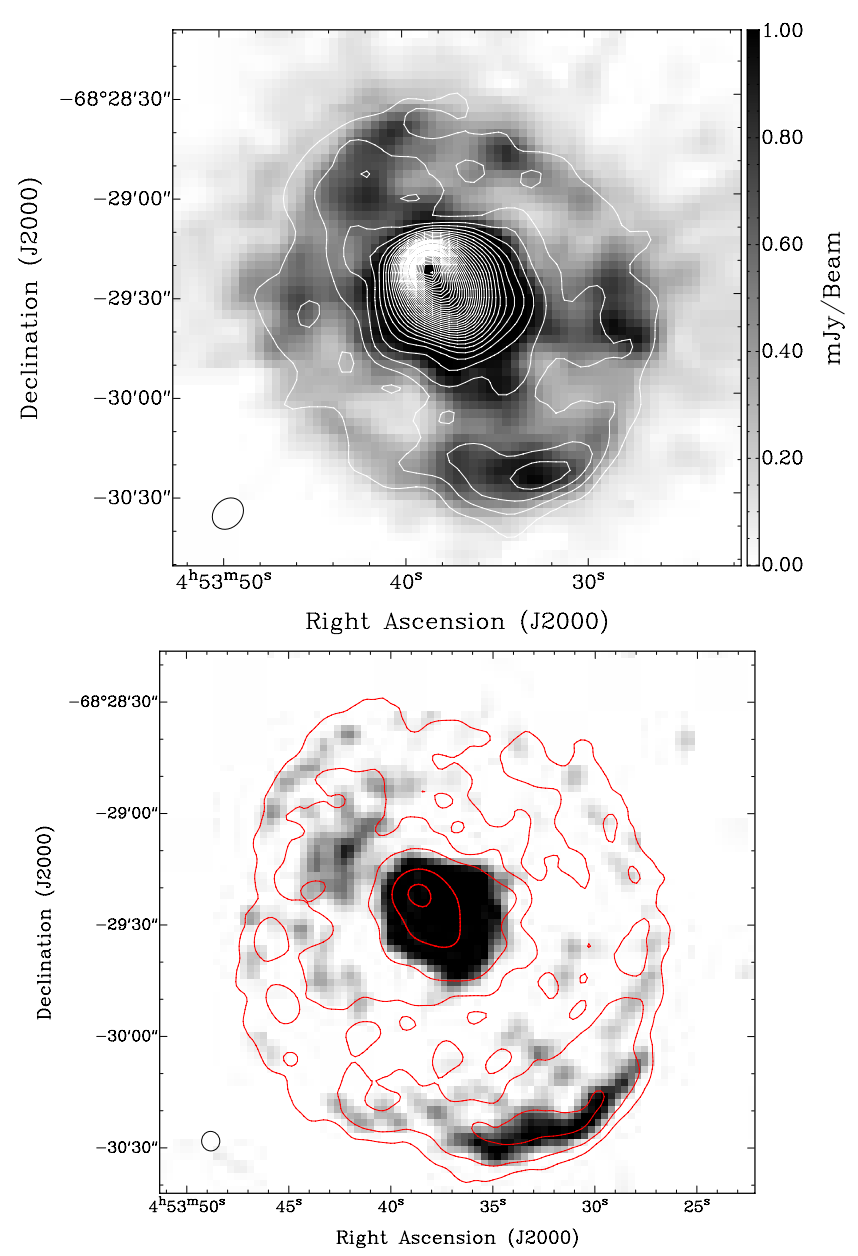

Fig. 1. ATCA observations of SNR J0453-6829. Top: $3 \mathrm{~cm}(8.6 \mathrm{GHz})$ image overlaid with $6 \mathrm{~cm}(4.8 \mathrm{GHz})$ contours. The contours are from 0.3 to $12.0 \mathrm{mJy} / \mathrm{beam}$ in steps of $0.3 \mathrm{mJy} /$ beam with a beam size of $10.3^{\prime \prime} \times 8.4^{\prime \prime}$. Bottom: $20 \mathrm{~cm}(1.4 \mathrm{GHz})$ image overlaid with $13 \mathrm{~cm}$ $(2.4 \mathrm{GHz})$ contours. The contours are $0.12,0.4,1,4$ and $8 \mathrm{mJy} / \mathrm{beam}$ with the $20 \mathrm{~cm}$ image beam size of $5.2^{\prime \prime} \times 4.9^{\prime \prime}$. Beam sizes are indicated in the bottom left corners.

image from Hughes et al. (2007). We took the $20 \mathrm{~cm}(1.4 \mathrm{GHz})$ and $13 \mathrm{~cm}(2.4 \mathrm{GHz})$ measurements from Gaensler et al. (2003) and added measurements at $20 \mathrm{~cm}(1.4 \mathrm{GHz})$ from the mosaics presented by Filipović et al. (2009) and Hughes et al. (2007). The main source of uncertainty in the measured flux densities is the definition of the area best representing the SNR, which dominates the statistical error. Based on different trials, we estimate that the combined flux density errors from all radio images used in this study (apart from $73 \mathrm{~cm}$ where the error is in order of 20\%; Mathewson et al. 1983; Clarke et al. 1976), are $\sim 10 \%$ at each given frequency. Using the flux densities from Table 1, we estimated spectral indices for the PWN $\left(\alpha_{\text {Core }}=-0.04 \pm 0.04\right)$, the outer SNR shell excluding the core $\left(\alpha_{\text {Shell }}=-0.43 \pm 0.01\right)$, and the whole SNR $\left(\alpha_{\text {Total }}=-0.39 \pm 0.03\right)$; see Fig. 2 .

In Fig. 3 we show the spatial distribution of the spectral index for SNR J0453-6829 derived from wavelengths of $20 \mathrm{~cm}$, $13 \mathrm{~cm}, 6 \mathrm{~cm}$, and $3 \mathrm{~cm}$. This image was formed by reprocessing all observations to a common $u-v$ range, and then fitting $S \propto v^{\alpha}$ pixel by pixel using all four images simultaneously. From this image, we can see that the vicinity around the central compact object exhibits relatively flat spectra with indices in the range $-0.2<\alpha<+0.2$, while the rest of the SNR exhibits spectra with $\alpha \sim-0.35$ to -0.7 , typical for a standard SNR. 
Table 1. Integrated flux density of SNR J0453-6829.

\begin{tabular}{lcccccccc}
\hline \hline $\begin{array}{l}v \\
(\mathrm{GHz})\end{array}$ & $\begin{array}{c}\lambda \\
(\mathrm{cm})\end{array}$ & $\begin{array}{c}\text { ATCA } \\
\text { Project }\end{array}$ & $\begin{array}{c}\text { rms } \\
(\mathrm{mJy})\end{array}$ & $\begin{array}{c}\text { Beam size } \\
\left({ }^{\prime \prime}\right)\end{array}$ & $\begin{array}{c}S_{\text {Core }} \\
(\mathrm{mJy})\end{array}$ & $\begin{array}{c}S_{\text {Shell }} \\
(\mathrm{mJy})\end{array}$ & $\begin{array}{c}S_{\text {Total }} \\
(\mathrm{mJy})\end{array}$ & Reference \\
\hline 0.408 & 73 & MOST & 50 & $157.3 \times 171.6$ & - & - & 350 & Mathewson et al. (1983) \\
0.843 & 36 & MOST & 0.4 & 43 & 57 & 171 & 228 & This work \\
1.388 & 20 & C373 & 1.2 & 40 & 45 & 140 & 185 & This work - MOSAIC \\
1.400 & 20 & C1074 & 0.1 & $7.3 \times 6.7$ & 46 & 140 & 186 & Gaensler et al. (2003) \\
1.400 & 20 & C1074, C373 & 0.05 & $5.2 \times 4.9$ & 46 & 140 & 185 & This work \\
2.400 & 13 & C1074 & 0.07 & $9.2 \times 8.4$ & 46 & 105 & 151 & Gaensler et al. (2003) \\
2.400 & 13 & C1074 & 0.04 & $7.8 \times 7.3$ & 48 & 105 & 153 & This work \\
4.790 & 6 & C634, C918, C1074 & 0.1 & $10.3 \times 8.4$ & 48 & 81 & 129 & This work \\
8.640 & 3 & C634, C918, C1074 & 0.1 & $10.3 \times 8.4$ & 46 & 64 & 100 & This work \\
\hline
\end{tabular}

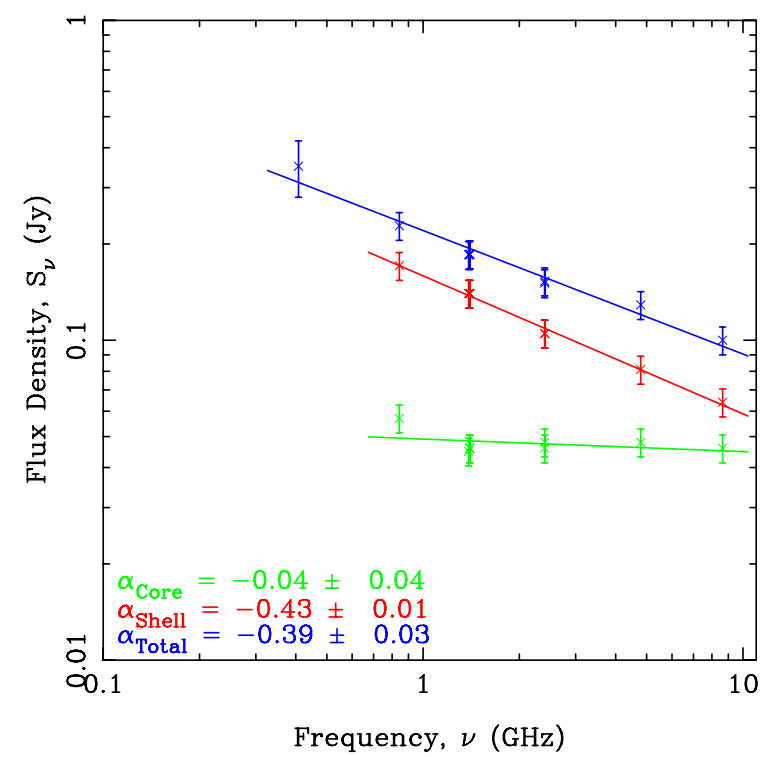

Fig. 2. Radio-continuum spectrum of SNR J0453-6829. The lines represent power-law fits to the flux densities of the core (green), shell (red) and combined core and shell (blue).

Linear polarisation images of SNR J0453-6829 at $6 \mathrm{~cm}$ and $3 \mathrm{~cm}$ were created using the $Q$ and $U$ Stokes parameters and are illustrated in Fig. 4. The fractional polarisation has been evaluated using the the standard MIRIAD task IMPOL. The majority of the polarised emission is seen in the core region. At $6 \mathrm{~cm}$, the SNR core shows a mean fractional polarisation of $\sim 7 \%$ whilst along the shell (at the southern side of the SNR), there is a pocket of quite strong uniform polarisation, with a mean value of $\sim 32 \%$ (Fig. 4, top) which corresponds to the observed total intensity brightening and possibly indicates varied dynamics along the shell. At $3 \mathrm{~cm}$ the mean fractional polarisation is $\sim 9 \%$. Our estimated peak value is $P \cong 44 \pm 12 \%$ at $6 \mathrm{~cm}$ and $12 \pm 6 \%$ at $3 \mathrm{~cm}$.

\subsection{X-rays}

SNR J0453-6829 was observed with the instruments of the XMM-Newton satellite. The observation (Obs. Id. 0062340101) was performed in March 2001 and here we present the results of the analysis of the X-ray data obtained by the European Photon Imaging Cameras (EPIC) and the Reflection Grating Spectrometers (RGS). The EPIC instruments were operated in full imaging modes using the medium optical blocking filters. Using the EPIC MOS1, MOS2 and pn CCDs yields sensitive X-ray observations of a $\sim 30^{\prime}$ field in the $0.15-10 \mathrm{keV}$

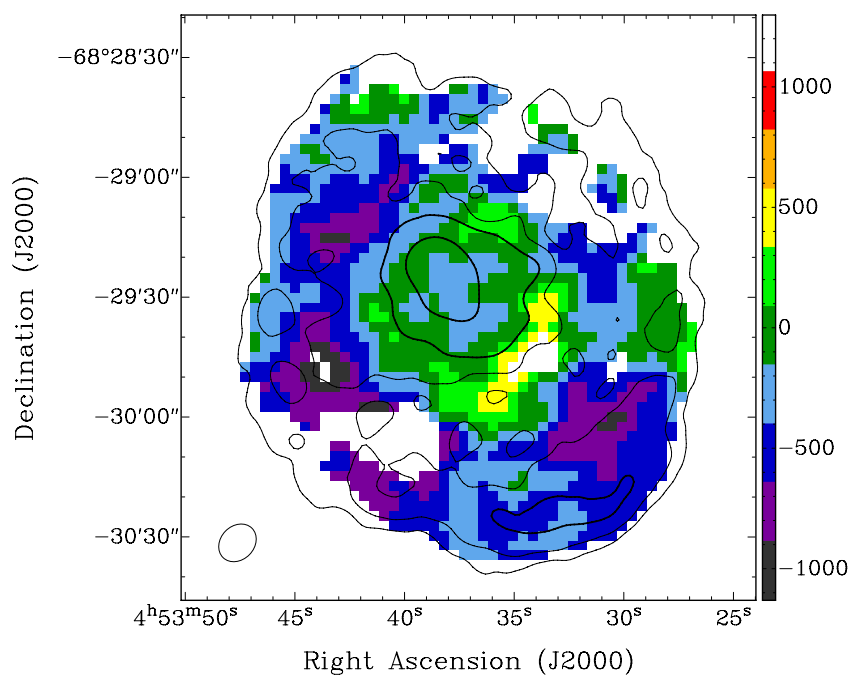

Fig. 3. Spectral index map of SNR J0453-6829 using wavelengths of $3 \mathrm{~cm}, 6 \mathrm{~cm}, 13 \mathrm{~cm}$, and $20 \mathrm{~cm}$. The sidebar gives the spectral index scale, e.g. -500 corresponds to $\alpha=-0.5$. Contours (from $13 \mathrm{~cm}$ ) are $0.12,0.4,1$ and $4 \mathrm{mJy} / \mathrm{beam}$. The beam size of $10.3^{\prime \prime} \times 8.4^{\prime \prime}$ is indicated in the bottom left corner.

energy band. Technical descriptions of the EPIC cameras can be found in Turner et al. (2001) and Strüder et al. (2001). The two RGS provide high-resolution X-ray spectra in the $0.3-2 \mathrm{keV}$ band (den Herder et al. 2001). A summary of the observations with the instrumental setups is given in Table 2. The XMM-Newton data were analysed with SAS v10.0.0 ${ }^{1}$. The data were affected by background flaring activity of moderate intensity. To produce images and spectra from the EPIC data we therefore removed intervals of high background while for the RGS the background level was below the recommended threshold ( $2 \mathrm{cts} \mathrm{s}^{-1}$ in CCD number 9 of the instrument) and we used the full exposure time for spectral extraction. The final net exposure times used for spectral analysis are listed in Table 2. For the EPIC spectra we extracted events subject to the canonical set of valid pixel patterns ( 0 to 12 for MOS; 0 to 4 for pn) and rejecting events from pixels flagged as bad. SNR J0453-6829 is of roughly circular shape with a diameter of about 2' (Gaensler et al. 2003), which causes a broadening of emission lines in the RGS spectra. This was taken into account when creating the response files with the task RGSRMFGEN by supplying the intensity profile of the remnant in the direction of the dispersion axis. This was created from an EPIC-pn image in the $0.2-2.0 \mathrm{keV}$

\footnotetext{
1 Science Analysis Software (SAS): http://xmm.esac.esa.int/sas/
} 


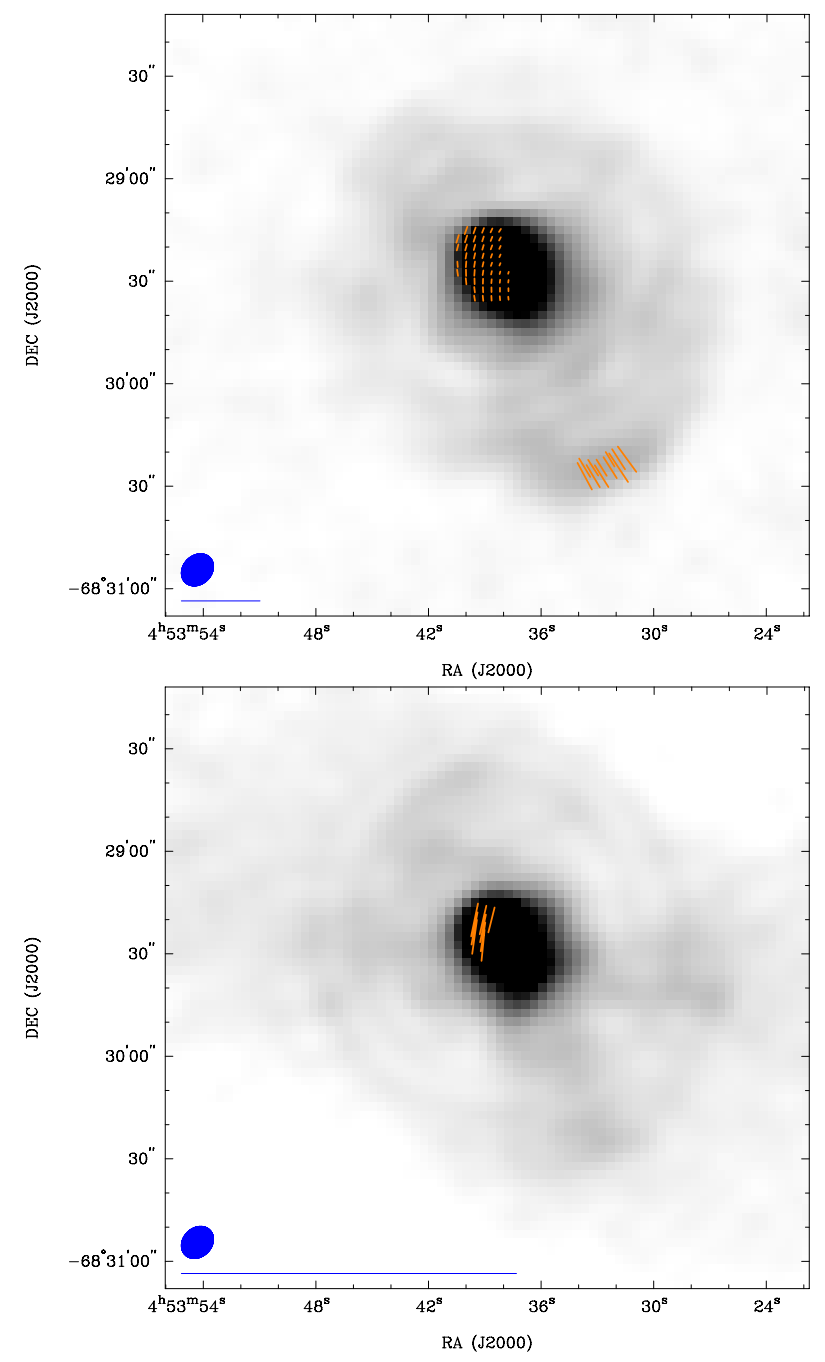

Fig. 4. Radio observations of SNR J0453-6829 at $6 \mathrm{~cm}$ (top) and $3 \mathrm{~cm}$ (bottom) matched to the $6 \mathrm{~cm}$ resolution. The blue circle in the lower left corner of the images represents the synthesised beam width of $10.3^{\prime \prime} \times 8.4^{\prime \prime}$. The length of the vectors represent the fractional polarised intensity at each position, and the orientation of the vectors indicates the mean position angle of the electric field (averaged over the observing bandwidth not corrected for Faraday rotation). The blue line below each circle represents the length of a polarisation vector of $100 \%$.

band. A colour image produced from the combined EPIC data is shown in Fig. 5. The PWN at the centre of the SNR cannot be resolved by the XMM-Newton instruments. Therefore, we extracted X-ray spectra from the full emission region of the remnant, including the PWN, and modelled the emission from the SNR and the PWN with different emission components.

All XMM-Newton spectra were modelled simultaneously using XSPEC (Arnaud 1996) version 12.7.0u, only allowing one relative normalisation factor for each instrument (using the EPIC pn spectrum as a reference with the factor fixed at 1.0). We fitted two-component models comprising of a thermal plasma emission and a power-law component, representing the thermal $\mathrm{X}$-ray emission from the SNR and the harder, non-thermal emission from the PWN, respectively. For the plasma emission we used different models available in XSPEC and report here on the results for the fits using either the plane-parallel shock (vpshock) model or the Sedov (vsedov) model (Borkowski et al. 2001). For both cases we used NEI-version 2.0 and allowed some chemical elemental abundances to vary in the fit. For elements which do
Table 2. XMM-Newton observation of SNR J0453-6829 on 2001-03-29.

\begin{tabular}{lccc}
\hline \hline $\begin{array}{l}\text { Instrument }^{a} \\
\text { configuration }\end{array}$ & $\begin{array}{c}\text { Start time } \\
\text { (UT) }\end{array}$ & $\begin{array}{c}\text { End time } \\
\text { (UT) }\end{array}$ & $\begin{array}{c}\text { Exposure }^{b} \\
\text { (s) }\end{array}$ \\
\hline EPIC MOS1 FF & $11: 47: 15$ & $17: 39: 19$ & 7366 \\
EPIC MOS2 FF & $11: 47: 15$ & $17: 39: 19$ & 7370 \\
EPIC pn eFF & $13: 00: 34$ & $17: 40: 02$ & 3619 \\
RGS1 Spectro & $11: 40: 55$ & $17: 41: 01$ & 20820 \\
RGS2 Spectro & $11: 40: 55$ & $17: 41: 01$ & 20440 \\
\hline
\end{tabular}

Notes. ${ }^{(a)}$ FF: full frame CCD readout mode with 2.6 s for MOS; EFF: pn "extended FF" with $200 \mathrm{~ms}$ frame time; ${ }^{(b)}$ net exposure time used for spectral analysis after removing intervals of high background.

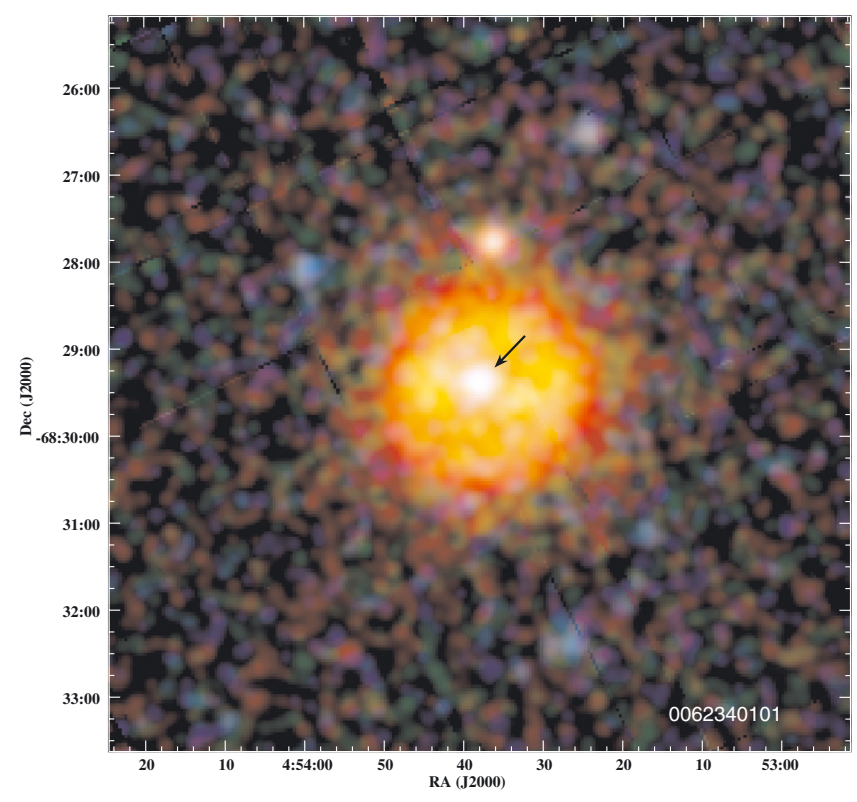

Fig. 5. Combined EPIC colour image of the field around SNR J0453-6829. Red, green, and blue colours denote X-ray intensities in the $0.2-1.0,1.0-2.0$ and $2.0-4.5 \mathrm{keV}$ bands. The strong soft X-ray emission from the SNR shell appears in yellow, while the bright white spot in the centre of the SNR (marked with a black arrow) is caused by the emission from the PWN. Unrelated point sources are seen around the remnant.

not contribute strongly to the X-ray spectra, the abundance was fixed to 0.5 solar, as is typical in the LMC (Russell \& Dopita 1992). The solar abundance table was taken from Wilms et al. (2000). Both, thermal and non-thermal emission components in the model were attenuated by two absorption components along the line of sight, one accounting for the Galactic foreground $a b-$ sorption with a column density of $6 \times 10^{20} \mathrm{~cm}^{-2}$ and solar abundances, and one for the absorption in the LMC with the column density as a free parameter and abundances set to 0.5 solar for all elements heavier than He. For the absorption and emission components in the LMC a radial velocity of $278 \mathrm{~km} \mathrm{~s}^{-1}$ (corresponding to a redshift of $9.27 \times 10^{-4}$ Richter et al. 1987) was adopted. The slope of the power-law was not well constrained in either model variant and we fixed the photon index at 2.0 (typical values for PWN are between 1.5 and 2.2 Kaspi et al. 2006). For the vpshock model we also fixed the lower parameter $\tau_{1}$ of the ionisation time-scale range $\tau_{1}<\tau_{\mathrm{u}}$ at $10^{8} \mathrm{~s} \mathrm{~cm}^{-3}$. The mean shock temperature and the electron temperature immediately behind the shock front can both be fitted by the Sedov model. First trial fits resulted in values fully consistent within their errors 


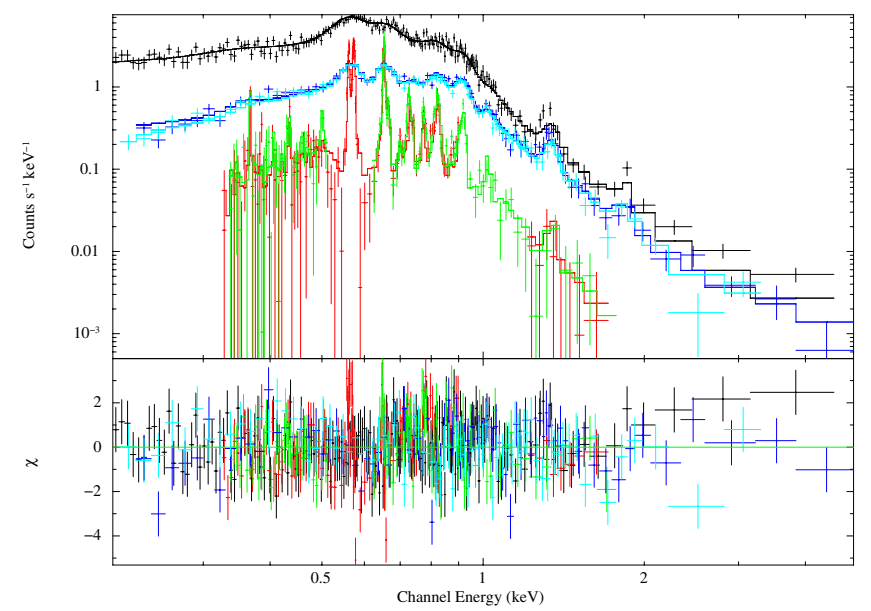

Fig. 6. Combined fit to the EPIC (pn: black, MOS1: blue, MOS2: light blue) and RGS (red: RGS1, green: RGS2) spectra of SNR J0453-6829. The data are denoted by the crosses with error bars and the histograms represent the best-fit model. In the lower panel the residuals are plotted in units of $\sigma$. The spectral extraction includes the emission from the SNR shell and the PWN and the model consists of thermal plasma emission (Sedov model) from the shell and a power-law from the PWN.

Table 3. Spectral fits to the XMM-Newton spectra of SNR J0453-6829 including its PWN.

\begin{tabular}{|c|c|c|}
\hline \multicolumn{2}{|c|}{ Model vpshock $\left(\chi_{\mathrm{r}}^{2}=1.48\right.$ for 574 d.o.f. $)$} & \multirow[b]{2}{*}{ Value $^{b}$} \\
\hline Component & Parameter & \\
\hline $\begin{array}{l}\text { LMC absorption } \\
\text { shock temperature } \\
\text { abundance }^{a} \\
\text { abundance } \\
\text { abundance } \\
\text { abundance } \\
\text { abundance } \\
\text { abundance } \\
\text { ionisation time scale }\end{array}$ & $\begin{array}{c}N_{\mathrm{H}}\left(10^{21} \mathrm{~cm}^{-2}\right) \\
k T(\mathrm{eV}) \\
\mathrm{N} \\
\mathrm{O} \\
\mathrm{Ne} \\
\mathrm{Mg} \\
\mathrm{Si} \\
\mathrm{Fe} \\
\tau_{\mathrm{u}}\left(10^{11} \mathrm{~s} \mathrm{~cm}^{-3}\right) \\
\end{array}$ & $\begin{array}{c}1.52(1.39-1.69) \\
250(234-269) \\
0.052(0.029-0.073) \\
0.104(0.094-0.113) \\
0.17(0.15-0.19) \\
0.31(0.25-0.36) \\
0.85(0.54-1.18) \\
0.20(0.18-0.23) \\
7.0(5.0-9.0) \\
\end{array}$ \\
\hline \multicolumn{3}{|c|}{ Model vsedov $\left(\chi_{\mathrm{r}}^{2}=1.33\right.$ for 574 d.o.f. $)$} \\
\hline $\begin{array}{l}\text { LMC absorption } \\
\text { shock/electron temp. } \\
\text { abundance } \\
\text { abundance } \\
\text { abundance } \\
\text { abundance } \\
\text { abundance } \\
\text { abundance } \\
\text { ionisation time scale }\end{array}$ & $\begin{array}{c}N_{\mathrm{H}}\left(10^{21} \mathrm{~cm}^{-2}\right) \\
k T(\mathrm{eV}) \\
\mathrm{N} \\
\mathrm{O} \\
\mathrm{Ne} \\
\mathrm{Mg} \\
\mathrm{Si} \\
\mathrm{Fe} \\
\tau\left(10^{13} \mathrm{~s} \mathrm{~cm}^{-3}\right)\end{array}$ & $\begin{array}{c}1.05(0.98-1.17) \\
168(164-173) \\
0.093(0.057-0.133) \\
0.20(0.17-0.23) \\
0.33(0.28-0.39) \\
0.52(0.42-0.62) \\
0.81(0.50-1.14) \\
0.39(0.34-0.44) \\
2.4(>1.0)\end{array}$ \\
\hline
\end{tabular}

Notes. ${ }^{(a)}$ Elemental abundances are specified relative to solar (Wilms et al. 2000). (b) $90 \%$ confidence ranges for one parameter of interest are given in parenthesis.

and in the following fits we forced the two temperatures to be the same.

Both models have the same number of degrees of freedom (d.o.f.) and the Sedov model yields a formally better fit (see Table 3). The XMM-Newton spectra, with the bestfit Sedov model, are shown in Fig. 6. The best fit parameters, which were free in the fit, are summarised in Table 3 for both models. The total observed flux in the $0.2-10 \mathrm{keV}$ band is $5.2 \times 10^{-12} \mathrm{erg} \mathrm{cm}^{-2} \mathrm{~s}^{-1}$ with a $12.0 \%$ contribution of the

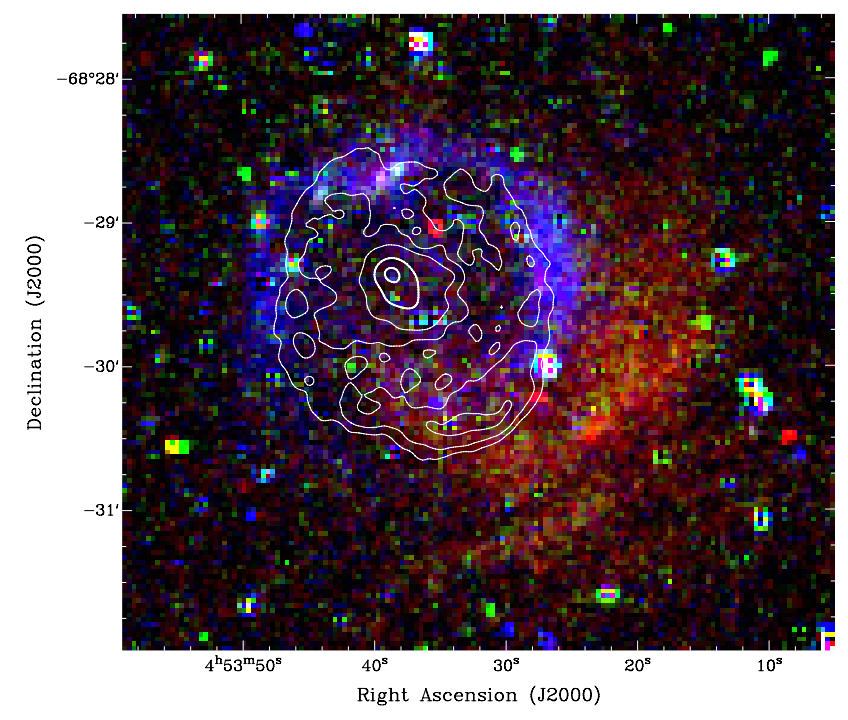

Fig. 7. MCELS composite optical image ( $\mathrm{RGB}=\mathrm{H}_{\alpha}$, [SII], [OIII]) of SNR J0453-6829 overlaid with $13 \mathrm{~cm}$ contours. The contours are 0.12 , $0.4,1,4$ and $8 \mathrm{mJy} / \mathrm{beam}$.

power-law (derived from the EPIC-pn spectrum for the Sedov model). This converts to an absorption corrected luminosity of $7.0 \times 10^{37} \mathrm{erg} \mathrm{s}^{-1}$.

\subsection{Optical}

The Magellanic Cloud Emission Line Survey (MCELS) ${ }^{2}$ was carried out at the $0.6 \mathrm{~m}$ University of Michigan/CTIO Curtis Schmidt telescope, equipped with a SITE $2048 \times$ $2048 \mathrm{CCD}$, which gave a field of $1.35^{\circ} \times 1.35^{\circ}$ at a scale of $2.4^{\prime \prime} \times 2.4^{\prime \prime}$ pixel $^{-1}$ (Smith et al. 2006). Both the LMC and SMC were mapped in narrow bands corresponding to $\mathrm{H} \alpha$, [O III] $(\lambda=5007 \AA)$, and $\left[\mathrm{S}_{\mathrm{II}}\right](\lambda=6716,6731 \AA)$, plus matched red and green continuum bands. All the data have been continuum subtracted, flux-calibrated and assembled into mosaic images. The region around SNR J0453-6829 is shown in Fig. 7 which demonstrates that [OIII] emission dominates around the outer edge of the SNR. We also note the enhanced $\mathrm{H}_{\alpha}$ emission in the south-west direction adjacent to the SNR.

\section{Discussion}

The SNR J0453-6829 in the LMC shows a prominent central core in radio and X-ray images, which is interpreted as a PWN (Gaensler et al. 2003). The PWN is surrounded by a slightly elongated shell centred at $\mathrm{RA}(\mathrm{J} 2000)=4^{\mathrm{h}} 53^{\mathrm{m}} 37^{\mathrm{s}} .2$, $\operatorname{Dec}(\mathrm{J} 2000)=-68^{\circ} 29^{\prime} 30^{\prime \prime}$ for which we derive a size of $\left(128^{\prime \prime} \pm 4^{\prime \prime}\right) \times\left(120^{\prime \prime} \pm 4^{\prime \prime}\right)$ (corresponding to $(31 \pm 1 \mathrm{pc}) \times$ $(29 \pm 1 \mathrm{pc})$ at a distance of $50 \mathrm{kpc})$ from the $13 \mathrm{~cm}$ radio data (obtained at position angles of $20^{\circ}$ and $110^{\circ}$, running from north to east). The shell radio emission shows brightening along its southern rim. Our size estimate is in agreement with the diameter previously reported from radio data by Gaensler et al. (2003). From the intensity profile of the EPIC data we estimate a size of $\sim 140^{\prime \prime}$ (roughly in east-west direction, at $10 \%$ of maximum intensity), which corresponds to $\sim 130^{\prime \prime}$ corrected for the angular resolution of the telescope. For a better estimate we re-analysed the Chandra data from 2001 presented by (Gaensler et al. 2003). From the Chandra image we measure $128^{\prime \prime} \pm 4^{\prime \prime}(31 \pm 1 \mathrm{pc})$ as the

${ }^{2}$ MCELS: http://www.ctio.noao.edu/mcels/ 


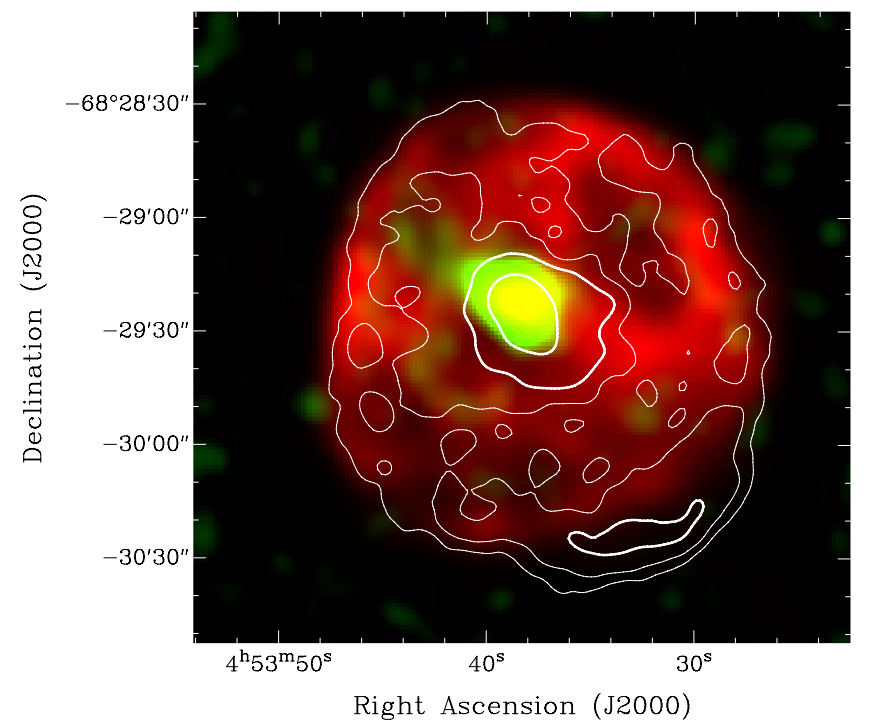

Fig. 8. Chandra colour image of SNR J0453-6829 in the $0.3-2.0 \mathrm{keV}$ (red) and 2.0-4.0 keV (green) energy bands overlaid with $13 \mathrm{~cm}$ contours. The contours are $0.12,0.4,1$ and $4 \mathrm{mJy} / \mathrm{beam}$ and the Chandra image was smoothed to the same resolution as the radio image.

largest extent, which is consistent with the radio extent (Fig. 8). So in the following considerations we assume a radius of $15.5 \mathrm{pc}$.

To estimate the age of the SNR from the vpshock model we used the relation $t_{\mathrm{y}}=3.8 \times 10^{2} R_{\mathrm{pc}}(k T)_{\mathrm{keV}}^{-1 / 2}$ from Xu et al. (2005) and assume the temperature derived from X-ray spectral modelling $(0.25 \mathrm{keV})$ and the radius of the SNR, which results in $\sim 11.8 \mathrm{kyr}$, consistent with the age inferred by Gaensler et al. (2003) from a preliminary X-ray spectral modelling. Also using a similar Sedov model and following Owen et al. (2011) who applied it to the spectrum of the SMC SNR IKT 16, we derive - with a shock temperature of $0.17 \mathrm{keV}$, and the normalisation of the Sedov model fit of $3.74 \times 10^{-2} \mathrm{~cm}^{-5}-$ the following physical parameters for SNR J0453-6829: electron density in the X-ray emitting material of $1.56 \mathrm{~cm}^{-3}$, dynamical age of $15.2 \mathrm{kyr}$, swept-up mass of $830 M_{\odot}$ and an explosion energy of $7.6 \times 10^{50} \mathrm{erg}$. Our estimate for the density is higher than that derived by Hughes et al. (1998) from ASCA observations (without knowledge of the PWN contributing to the X-ray spectrum of the remnant) and consistent with the average value of the other LMC SNRs in that work. This directly reflects the higher density of the ISM in the LMC in comparison to the SMC (van der Heyden et al. 2004).

The shock temperature (and electron temperature in our Sedov modelling) is very low compared to other remnants in the MCs. The whole SMC sample modelled by van der Heyden et al. (2004) and Filipović et al. (2008) in a similar way exhibits temperatures between $0.26 \mathrm{keV}$ and $1.8 \mathrm{keV}$. The low temperature leads to a relatively high dynamical age for SNR J0453-6829. The high swept-up mass is also consistent with an SNR well evolved into its Sedov phase. As discussed in van der Heyden et al. (2004) the higher ISM density and higher abundances in the LMC compared to the SMC can lead to a faster evolution to the radiative cooling stage.

The abundances derived from our spectral analysis exhibit some model dependences, but are generally consistent or somewhat lower than average abundances in the ISM of the LMC. The only exception is $\mathrm{Si}$ which is overabundant with respect to the other elements. This suggests that the X-ray spectrum is dominated by emission from swept-up ISM material, which makes it difficult to draw conclusions on the type of supernova explosion from abundance measurements. In the case of SNR J0453-6829 the association of the PWN with the remnant and its morphology favour a core-collapse supernova (Lopez et al. 2009).

The radio spectral index of $\alpha_{\text {Shell }}=-0.43$, confirms the nonthermal nature of the SNR shell emission in the radio band, while the flat spectral index for the core of $\alpha_{\text {Core }}=-0.04$ is typical for a PWN. The overall spectral index of $\alpha=-0.39$ is slightly "flatter" in comparison with typical values of -0.5 for SNRs (Mathewson et al. 1985; Filipović et al. 1998a). The radio spectra definitely confirm the PWN nature of the central object. At higher frequencies (Fig. 2) the flux density decreases as expected whereas at lower frequencies non-thermal radiation of the shell dominates. Overall, the radio-continuum properties of SNR J0453-6829 are very similar to SNR B0540-693, the most prominent SNR with a PWN in the LMC (Manchester et al. 1993).

Mathewson et al. (1983) found distinctive optical connections to X-ray and radio features of this SNR. As can be seen in the MCELS images (Fig. 7), the [O III] emission dominates in the outer parts of the remnant. This could indicate an oxygenrich type of SNR and suggests a type Ib SN event - the explosion of a massive O, B, or WR star (Arbutina \& Urošević 2005). We also note that prominent $\mathrm{H} \alpha$ emission is visible in the southwest, which might suggest that higher density ISM material is causing the brightening in the radio shell emission at the outer rim of the SNR in this direction. While no molecular clouds are reported in this region (Fukui et al. 2008; Kawamura et al. 2009), we do note that SNR J0453-6829 lies in a region of low H I column density with the rim density highest in the south west (Kim et al. 1998, 1999). The radio spectral index (Fig. 3) in this southwest region indicates non-thermal emission $(\alpha \sim-0.4)$. Careful comparison (after correcting for the rotated presentation) of the 24 and $70 \mu \mathrm{m}$ infra-red data shows no correspondence between the enhanced background reported by Williams et al. (2006) and SNR J0453-6829. In contrast however, the Chandra image reveals only weak X-ray emission at the location of the bright radio feature. This could be explained by the higher density material being located in front of the rim of the SNR, suppressing the $\mathrm{X}$-rays, or, together with the high polarisation of the $6 \mathrm{~cm}$ radio emission in this area, might favour a locally increased magnetic field strength enhancing the non-thermal radio emission. Future spatially resolved X-ray spectroscopy should be able to resolve this question.

Acknowledgements. This publication is partly based on observations with $X M M-N e w t o n$, an ESA Science Mission with instruments and contributions directly funded by ESA Member states and the USA (NASA). The XMM-Newton project is supported by the Bundesministerium für Wirtschaft und Technologie/Deutsches Zentrum für Luft- und Raumfahrt (BMWI/DLR, FKZ 50 OX 0001) and the Max-Planck Society. We used the KARMA software package developed by the ATNF. The Australia Telescope Compact Array is part of the Australia Telescope which is funded by the Commonwealth of Australia for operation as a National Facility managed by CSIRO. The Magellanic Clouds Emission Line Survey (MCELS) data are provided by R. C. Smith, P. F. Winkler, and S. D. Points. The MCELS project has been supported in part by NSF grants AST-9540747 and AST-0307613, and through the generous support of the Dean B. McLaughlin Fund at the University of Michigan, a bequest from the family of Dr. Dean B. McLaughlin in memory of his lasting impact on Astronomy. The National Optical Astronomy Observatory is operated by the Association of Universities for Research in Astronomy Inc. (AURA), under a cooperative agreement with the National Science Foundation.

\section{References}

Arbutina, B., \& Urošević, D. 2005, MNRAS, 360, 76

Arnaud, K. A. 1996, in Astronomical Data Analysis Software and Systems V, ASP Conf. Ser., 101, 17 
Blair, W. P., Ghavamian, P., Sankrit, R., \& Danforth, C. W. 2006, ApJS, 165, 480 Bojičić, I. S., Filipović, M. D., Parker, Q. A., et al. 2007, MNRAS, 378, 1237 Borkowski, K. J., Lyerly, W. J., \& Reynolds, S. P. 2001, ApJ, 548, 820

Bozzetto, L. M., Filipović, M. D., Crawford, E. J., et al. 2010, Serbian Astron. J., 181, 43

Bozzetto, L. M., Filipović, M. D., Crawford, E. J., et al. 2012a, MNRAS, 420, 2588

Bozzetto, L. M., Filipovic, M. D., Crawford, E. J., et al. 2012b, Rev. Mex. Astron. Astrofis., 48, 41

Čajko, K. O., Crawford, E. J., \& Filipović, M. D. 2009, Serbian Astron. J., 179, 55

Clarke, J. N., Little, A. G., \& Mills, B. Y. 1976, Aust. J. Phys. Astrophys. Suppl., 40,1

Crawford, E. J., Filipović, M. D., de Horta, A. Y., Stootman, F. H., \& Payne, J. L. 2008a, Serbian Astron. J., 177, 61

Crawford, E. J., Filipovic, M. D., \& Payne, J. L. 2008b, Serbian Astron. J., 176, 59

Crawford, E. J., Filipović, M. D., Haberl, F., et al. 2010, A\&A, 518, A35

den Herder, J. W., Brinkman, A. C., Kahn, S. M., et al. 2001, A\&A, 365, L7 di Benedetto, G. P. 2008, MNRAS, 390, 1762

Dickel, J. R., Gruendl, R. A., McIntyre, V. J., \& Amy, S. W. 2010, AJ, 140, 1511

Filipović, M. D., Haynes, R. F., White, G. L., \& Jones, P. A. 1998a, A\&AS, 130, 421

Filipović, M. D., Pietsch, W., Haynes, R. F., et al. 1998b, A\&AS, 127, 119

Filipović, M. D., Haberl, F., Winkler, P. F., et al. 2008, A\&A, 485, 63

Filipović, M. D., Crawford, E. J., Hughes, A., et al. 2009, in IAU Symp. 256, eds. J. T. van Loon, \& J. M. Oliveira, 8

Fukui, Y., Kawamura, A., Minamidani, T., et al. 2008, ApJS, 178, 56

Gaensler, B. M., Hendrick, S. P., Reynolds, S. P., \& Borkowski, K. J. 2003, ApJ, 594, L111

Gooch, R. 1996, in Astronomical Data Analysis Software and Systems V, eds. G. H. Jacoby, \& J. Barnes, ASP Conf. Ser., 101, 80

Haberl, F., \& Pietsch, W. 1999, A\&AS, 139, 277

Haynes, R. F., Klein, U., Wayte, S. R., et al. 1991, A\&A, 252, 475

Hughes, J. P., Hayashi, I., \& Koyama, K. 1998, ApJ, 505, 732
Hughes, A., Staveley-Smith, L., Kim, S., Wolleben, M., \& Filipović, M. 2007, MNRAS, 382, 543

Kaspi, V. M., Roberts, M. S. E., \& Harding, A. K. 2006, Isolated neutron stars, eds. W. H. G. Lewin, \& M. van der Klis, 279

Kawamura, A., Mizuno, Y., Minamidani, T., et al. 2009, ApJS, 184, 1

Kim, S., Staveley-Smith, L., Dopita, M. A., et al. 1998, ApJ, 503, 674

Kim, S., Dopita, M. A., Staveley-Smith, L., \& Bessell, M. S. 1999, AJ, 118, 2797

Long, K. S., Helfand, D. J., \& Grabelsky, D. A. 1981, ApJ, 248, 925

Lopez, L. A., Ramirez-Ruiz, E., Badenes, C., et al. 2009, ApJ, 706, L106

Manchester, R. N., Staveley-Smith, L., \& Kesteven, M. J. 1993, ApJ, 411, 756

Manchester, R. N., Fan, G., Lyne, A. G., Kaspi, V. M., \& Crawford, F. 2006, ApJ, 649, 235

Mathewson, D. S., Ford, V. L., Dopita, M. A., et al. 1983, ApJS, 51, 345

Mathewson, D. S., Ford, V. L., Tuohy, I. R., et al. 1985, ApJS, 58, 197

Mills, B. Y., Turtle, A. J., Little, A. G., \& Durdin, J. M. 1984, Aust. J. Phys., 37, 321

Owen, R. A., Filipović, M. D., Ballet, J., et al. 2011, A\&A, 530, A132

Payne, J. L., White, G. L., \& Filipović, M. D. 2008, MNRAS, 383, 1175

Richter, O.-G., Tammann, G. A., \& Huchtmeier, W. K. 1987, A\&A, 171, 33

Russell, S. C., \& Dopita, M. A. 1992, ApJ, 384, 508

Sasaki, M., Haberl, F., \& Pietsch, W. 2000, A\&AS, 143, 391

Sault, R. J., \& Wieringa, M. H. 1994, A\&AS, 108, 585

Sault, R. J., Teuben, P. J., \& Wright, M. C. H. 1995, in Astronomical Data Analysis Software and Systems IV, eds. R. A. Shaw, H. E. Payne, \& J. J. E. Hayes, ASP Conf. Ser., 77, 433

Smith, C., Points, S., \& Winkler, P. F. 2006, NOAO Newsletter, 85, 6

Strüder, L., Briel, U., Dennerl, K., et al. 2001, A\&A, 365, L18

Turner, M. J. L., Abbey, A., Arnaud, M., et al. 2001, A\&A, 365, L27

van der Heyden, K. J., Bleeker, J. A. M., \& Kaastra, J. S. 2004, A\&A, 421, 1031

Wang, Q., Hamilton, T., Helfand, D. J., \& Wu, X. 1991, ApJ, 374, 475

Williams, R. M., Chu, Y.-H., Dickel, J. R., et al. 1999, ApJS, 123, 467

Williams, B. J., Borkowski, K. J., Reynolds, S. P., et al. 2006, ApJ, 652, L33

Wilms, J., Allen, A., \& McCray, R. 2000, ApJ, 542, 914

Xu, J.-W., Zhang, X.-Z., \& Han, J.-L. 2005, Chin. J. Astron. Astrophys., 5, 165 